\title{
Inclusión del yogurt artesanal de leche de búfala en el pienso de gallinas ponedoras Isa Brown y su efecto en la producción y calidad del huevo
}

\section{Inclusion of buffalo milk artisan yogurt in the feed for laying hens Isa Brown and its effect on egg production and quality}

\author{
Abel Ortiz-Milán' \\ Fecha de recepción: 1 de marzo de 2016 \\ Fecha de aprobación: 2 de diciembre de 2016 \\ Solimar Gómez-Sarabia² \\ Osmany Jay-Herrera ${ }^{3}$ \\ Odelin Brea-Maura ${ }^{4}$
}

\begin{abstract}
Resumen
Con el objetivo de evaluar la inclusión de 0 (control), 5, 10 y $15 \mathrm{ml}$ de yogurt artesanal/ave/día en el pienso de gallinas ponedoras Isa Brown, y su efecto en la producción y calidad del huevo, se utilizaron 240 aves durante 60 días, en el estado Delta Amacuro, Venezuela, siguiendo un diseño completamente aleatorizado, con cuatro tratamientos y seis repeticiones; cada repetición se realizó con 10 gallinas. Al final del experimento se obtuvo que la producción total de huevos $(3.04,3.22,3.38$ y $3.58 \mathrm{~kg} / \mathrm{ave})$, el índice de puesta $(88.66,90.00,93.33$ y $96.66 \%)$, la conversión $(2.37,2.24,2.13$ y 2.01 kg alimento/ $\mathrm{kg}$ huevo) y el peso del huevo $(58.96,60.86,62.33$ y $65.26 \mathrm{~g}$ ) mejoraron significativamente en las aves a medida que se incrementó en el pienso la dosis de yogurt de búfala. El porciento de huevos rotos no difirió entre tratamientos, mientras que el porciento de huevos cascados y huevos sucios fue superior significativamente para $15 \mathrm{ml}$ de yogurt con respecto al control. Las características sensoriales del huevo cocido (olor, sabor y textura) no mostraron cambios significativos, según el criterio de los panelistas encuestados. Se concluye que la inclusión del yogurt artesanal elaborado con leche de búfala en la dieta de gallinas ponedoras Isa Brown estimuló el índice de puesta y el tamaño del huevo, sin que se presentaran efectos significativos en la calidad sensorial del huevo.
\end{abstract}

Palabras clave: aditivos; aves; Lactobacillus.

1 Ph. D. Universidad de Guantánamo (Guantánamo, Cuba). abel@cug.co.cu.

2 M. Sc. Universidad Territorial Deltaica Francisco Tamayo. (Tucupita, Venezuela).

3 Ph. D. Universidad de Guantánamo (Guantánamo, Cuba).

4 Ph. D. (c) Universidad de Matanzas Camilo Cienfuegos (Matanzas, Cuba). 


\begin{abstract}
A total of 240 birds were used in the state of Delta Amacuro, Venezuela, for 60 days in order to assess the inclusion of 0 (control), 5, 10 and $15 \mathrm{ml}$ of artisan yogurt / bird / day in the feed for laying hens Isa Brown, and its effect on egg production and quality, according to completely randomized design with four treatments and six repetitions, each repetition consisted of 10 hens. At the end of the experiment it was found that the total egg production $(3.04,3.22,3.38$ and $3.58 \mathrm{~kg} / \mathrm{bird})$, the laying rate $(88.66$, $90.00,93.33$ and $96.66 \%)$, conversion $(2.37,2.24,2.13$ and $2.01 \mathrm{~kg}$ feed/kg eggs) and egg weight (58.96, 60.86, 62.33 and $65.26 \mathrm{~g})$ improved significantly in birds to the extent that it was increased on feed the buffalo yogurt dose. The percentage of broken eggs did not differ between treatments, while in the percentage of eggs hulled and dirty eggs was significantly superior for $15 \mathrm{ml}$ of yogurt with respect control. The sensory characteristics of cooked egg (smell, taste and texture) showed no significant changes according to panelists' criteria. It is concluded that the inclusion of artisan yogurt made from buffalo milk in the diet for laying hens Isa Brown stimulated laying rate and egg size without significant effects in the sensory quality of the egg.
\end{abstract}

Keywords: additive; birds; Lactobacillus.

\title{
Cómo citar este artículo:
}

Ortiz-Milán A, Gómez-Sarabia S, Jay-Herrera O, Brea-Maura O. Inclusión del yogurt artesanal de leche de búfala en el pienso de gallinas ponedoras Isa Brown y su efecto en la producción y calidad del huevo. Rev. Cien. Agri. 2017; 14(1): 85-93. 


\section{Introducción}

La distribución de la masa bufalina en el mundo abarca todos los continentes, pero tiene mayor presencia en Asia (India, Pakistán, Tailandia, China, Vietnam), África (Egipto), Europa (Italia, Bulgaria) y América del Sur (Brasil, Colombia, Argentina, Venezuela y Perú); en general, la especie se cría para la producción multipropósito, con énfasis en la producción de leche y sus derivados (1).

Según Hurtado (2), el crecimiento de la ganadería bufalina en Venezuela y otros países del área ha sido impresionante en los últimos 25 años; los estados venezolanos de Monagas y Delta Amacuro, en el norte del país, se presentan como los mayores productores de leche de búfala, gracias a sus propicias condiciones climáticas y agroecológicas para el desarrollo de esta especie, las cuales favorecen un crecimiento importante de la producción y su aceptación por la población, sobre todo para la elaboración de quesos.

Desde principios del siglo $\mathrm{XX}$, el científico Metchnikoff (3) estudió las relaciones entre el consumo de cultivos lácteos fermentados con Lactobacillus y la baja incidencia de enfermedades y la longevidad de la población humana de Bulgaria. La investigación con los productos lácteos fermentados continuó hasta la década de los años treinta, cuando se descubrieron los primeros antibióticos y se marginó el trabajo con los lactobacillus, y se reactivó a principios de los años sesenta, cuando inició la crítica al uso de antibióticos como promotores de crecimiento del ganado, crítica que culminó con una legislación estricta que prohíbe su utilización en toda la Comunidad Europea y otros países de avanzada que se preocupan por sus efectos potencialmente peligrosos para la salud humana y la ecología (4 y 5).

El concepto del uso de los microorganismos que ayudan a la digestión, absorción y aprovechamiento de nutrientes y a la integridad y desarrollo de la mucosa intestinal, tanto en los humanos como en los animales, ha sido una inquietud científica y práctica (6 y 7). La elevada disponibilidad de leche de búfala en el norte de Venezuela, y su relativo bajo precio en comparación con aditivos comerciales con efecto probiótico, los cuales eran inasequibles para las pequeñas y medianas empresas, incitó a evaluar el yogurt artesanal elaborado con leche de búfala y su posible efecto en la producción y la calidad del huevo de gallinas ponedoras Isa Brown.

\section{Materiales y métodos}

\section{A. Ubicación y alojamiento de las aves}

La investigación se realizó en la Unidad de Gallinas Ponedoras del Fundo La Victoria, ubicado en el sector La Manaca, estado Delta Amacuro, Venezuela, en el período de enero a marzo de 2014. Se utilizaron durante 60 días un total de 240 aves de la raza Isa Brown, con un peso inicial aproximado de $1570 \mathrm{~g}$, lo que coincide con el pico de puesta ( 35 a 43 semanas de edad) y 18 horas de iluminación diarias.

Las aves se alojaron en jaulas metálicas de un metro de largo por $50 \mathrm{~cm}$. de ancho, divididas por la mitad. Se ubicaron 10 gallinas por jaula (5 por hueco) y se distribuyeron con un diseño completamente aleatorizado con cuatro tratamientos y seis repeticiones; se utilizaron 10 aves por repetición, para un total de 60 por tratamiento.

\section{B. Tratamientos e indicadores}

Los tratamientos consistieron en la inclusión de 0 $\mathrm{ml}$ (control sin yogurt), $5 \mathrm{ml}, 10 \mathrm{ml}$ y $15 \mathrm{ml}$ de yogurt líquido en el pienso/ave/día. Para determinar el efecto del yogurt se realizó análisis de varianza a las variables producción de huevo $(\mathrm{kg})$, peso del huevo al inicio y final del experimento (g), incremento en el peso del huevo (g) y conversión $(\mathrm{kg})$, mientras que para las variables índice de puesta/día (\%) y huevos cascados (\%), rotos (\%) y sucios $(\%)$ se empleó el método de comparación de proporciones.

En todos los casos la comparación post hoc de las medias se realizó a través de la prueba de rango múltiple de Duncan (8). Los programas utilizados en el análisis estadístico fueron el SPSS 15.0 (9), 
para el análisis de varianza, y el Comparpro 1.0 (10), para la comparación de proporciones.

Para el estudio de las características sensoriales del huevo, fueron cocidos sin adición de condimentos 12 huevos por tratamiento, y puestos al criterio de 12 panelistas especializados, sin hábitos de fumar ni de ingerir habitualmente café o bebidas alcohólicas, con condiciones de salud excelentes y edades entre 20 y 55 años. Los criterios de calidad sensorial encuestados fueron: olor (agradable, rancio u otros), sabor (normal, rancio y extraño) y textura (suave o fibrosa), según lo indicado por Schreiner et al. (11); además, se consideró el color de la yema, empleando el abanico Roche.

\section{Manejo de la alimentación y elaboración del yogurt}

Los comederos y bebederos en la nave experimental se distribuyeron de forma lineal al frente de las jaulas, lo que corresponde a $10 \mathrm{~cm}$. de frente de comederos y bebederos por ave. La composición y aporte del pienso comercial utilizado fue el mismo en todos los tratamientos (Tabla I), y se escogió teniendo en cuenta los requerimientos para gallinas ponedoras huevos marrones.

Las gallinas recibieron $120 \mathrm{~g}$ de pienso/ave/día en una comida que se ofreció a las 8:00 a.m. de cada día, mientras que el yogurt de búfala se adicionó sobre el pienso inmediatamente después de ofrecer este alimento, entre las 8:00 y las 8:30 a.m. de cada día.

Para elaborar el yogurt artesanal de leche de búfala se utilizó un inóculo compuesto por yogurt comercial de leche de vaca sin saborizante natural o artificial y sin azúcar, mientras que la leche de búfala contenía: $16.55 \%$ de sólidos totales, 7.32 \% de grasa, $3.89 \%$ de proteína, $4.45 \%$ de lactosa y $0.83 \%$ de cenizas, con un $\mathrm{pH}$ de 6.7 y una densidad de $1.0309 \mathrm{~g} / \mathrm{ml}$.

Se mezcló 1 litro de yogurt comercial por cada 20 litros de leche de búfala, a una temperatura de \pm $38{ }^{\circ} \mathrm{C}$; luego, se dejó en reposo durante 5 horas, aproximadamente, $\mathrm{y}$, posteriormente, se conservó en frío de 5 a $10^{\circ} \mathrm{C}$ hasta 7 días, máximo, después de elaborado.

Tabla I. Composición y aporte nutritivo del pienso para ponedora Isa Brown.

\begin{tabular}{|l|c|}
\hline \multicolumn{1}{|c|}{ Materias primas } & \% \\
\hline Harina de maíz & 58.84 \\
\hline Harina de soya & 29.00 \\
\hline Aceite vegetal & 2.00 \\
\hline Premezcla vitamínica & 1.00 \\
\hline Fosfato dicálcico & 1.00 \\
\hline Carbonato de calcio & 7.00 \\
\hline Sal común & 0.25 \\
\hline DL-Metionina & 0.75 \\
\hline Antioxidante Aportes & 0.01 \\
\hline Colina & 0.15 \\
\hline & \\
\hline Proteína bruta, \% & 17.40 \\
\hline Energía metabolizable, kcal/kg & 2870 \\
\hline Calcio, \% & 3.00 \\
\hline Fósforo total, \% & 0.50 \\
\hline Metionina, \% & 1.00 \\
\hline
\end{tabular}




\section{Resultados y discusión}

En la producción avícola es fundamental favorecer el desarrollo de la mucosa intestinal, así como mantener su integridad, pues este tejido es la vía de ingreso de los nutrientes $y$, por tanto, de la posibilidad de convertirlos en carne y huevo (12).

La Tabla II muestra cómo la producción total de huevos y el índice de puesta aumentaron significativamente $(P<0.05)$ en las aves a medida que se adicionó en el pienso el yogurt de búfala en dosis crecientes de 5, 10 y 15 ml/ave/día; en este sentido, la conversión también mostró significativamente los mejores índices en la medida que se incrementó el nivel de yogurt en el pienso de las aves. Tal comportamiento pudo deberse a que los lactobacillus y las levaduras son capaces de manifestar diferentes funciones a nivel del tracto gastrointestinal, creando un estado beneficioso para el resto del organismo. Al respecto, García (13) hace referencia a que las bacterias lácticas favorecen la supresión de microorganismos patógenos, ya que producen una serie de sustancias antimicrobianas, entre las que se encuentran el peróxido de hidrógeno $\left(\mathrm{H}_{2} \mathrm{O}_{2}\right)$, el diacetilo, la reuterina, los ácidos orgánicos, como el láctico y el acético, y las sustancias de naturaleza proteica conocidas como bacteriocinas.

Tabla II. Producción total de huevos, índice de puesta y conversión de alimentos en gallinas ponedoras, según el nivel de yogurt adicionado en el pienso.

\begin{tabular}{|l|c|c|c|c|c|c|}
\hline \multirow{2}{*}{ Indicadores } & \multicolumn{3}{|c|}{ Nivel de yogurt en el pienso/ave/día } & \multirow{2}{*}{ E.E \pm} & \multirow{2}{*}{ Sig. } \\
\cline { 2 - 5 } & $\mathbf{0}$ (control) & $\mathbf{5 ~} \mathbf{~} \mathbf{l}$ & $\mathbf{1 0} \mathbf{~} \mathbf{~}$ & $\mathbf{1 5} \mathbf{~} \mathbf{l}$ & & \\
\hline $\begin{array}{l}\text { Producción total de } \\
\text { huevos/ave, kg }\end{array}$ & $3.04^{\mathrm{a}}$ & $3.22^{\mathrm{b}}$ & $3.38^{\mathrm{c}}$ & $3.58^{\mathrm{d}}$ & 0.02 & $\mathrm{P}<0.05$ \\
\hline Índice de puesta, \% & $86.66^{\mathrm{a}}$ & $90.00^{\mathrm{b}}$ & $93.33^{\mathrm{c}}$ & $96.66^{\mathrm{d}}$ & 0.42 & $\mathrm{P}<0.05$ \\
\hline $\begin{array}{l}\text { Conversión, kg ali./kg } \\
\text { huevo }\end{array}$ & $2.37^{\mathrm{a}}$ & $2.24^{\mathrm{b}}$ & $2.13^{\mathrm{c}}$ & $2.01^{\mathrm{d}}$ & 0.01 & $\mathrm{P}<0.05$ \\
\hline $\begin{array}{l}\text { Conversión, g ali./ } \\
\text { huevo }\end{array}$ & $138.46^{\mathrm{a}}$ & $133.33^{\mathrm{b}}$ & $128.57^{\mathrm{c}}$ & $124.14^{\mathrm{d}}$ & 0.68 & $\mathrm{P}<0.05$ \\
\hline
\end{tabular}

abcd Medias con letras diferentes dentro de la misma fila difieren a $\mathrm{P}<0.05$ (8)

Por su parte, las levaduras sintetizan vitaminas del complejo B que estimulan el crecimiento de las poblaciones de lactobacillus y bacterias acetogénicas (14), lo que provoca una acción sinérgica y efectos beneficiosos para el huésped.

Según Gómez y Balcázar (15), en general, la incorporación de las bacterias lácticas, levaduras y otros microorganismos con efecto probiótico en la dieta potencian los mecanismos de defensa del intestino de los animales, por lo que pueden ser eficaces en la prevención, en la terapia de infecciones entéricas y en la inmunoterapia antitumor y antialérgica.

En la Tabla III se muestra un incremento significativo $(\mathrm{P}<0.05)$ del peso del huevo a las 43 semanas de edad de las aves, así como el incremento del peso del huevo en la medida que se incrementó el nivel de yogurt en las dietas de las aves. 
Tabla III. Indicadores relacionados con el peso del huevo según el nivel de yogurt adicionado en el pienso.

\begin{tabular}{|l|c|c|c|c|c|c|}
\hline \multirow{2}{*}{\multicolumn{1}{|c|}{ Indicadores }} & \multicolumn{5}{c|}{ Nivel de yogurt en el pienso/ave/día } & \multirow{2}{*}{ Sig. } \\
\cline { 2 - 6 } & $\begin{array}{c}\mathbf{0 ~} \mathbf{~ m l} \\
\text { (control) }\end{array}$ & $\mathbf{5 ~} \mathbf{~ m l}$ & $\mathbf{1 0} \mathbf{~ m l}$ & $\mathbf{1 5} \mathbf{~ m l}$ & E.E \pm & \\
\hline Peso del huevo semana 35, g & 58.13 & 58.45 & 58.35 & 58.20 & 0.12 & $\mathrm{P}=0.20$ \\
\hline Peso del huevo semana 43, g & $58.96^{\mathrm{a}}$ & $60.86^{\mathrm{b}}$ & $62.33^{\mathrm{c}}$ & $65.26^{\mathrm{d}}$ & 0.32 & $\mathrm{P}<0.05$ \\
\hline $\begin{array}{l}\text { Incremento de peso del huevo, } \\
\mathrm{g}\end{array}$ & $0.83^{\mathrm{a}}$ & $2.41^{\mathrm{b}}$ & $3.98^{\mathrm{c}}$ & $7.06^{\mathrm{d}}$ & 0.27 & $\mathrm{P}<0.05$ \\
\hline
\end{tabular}

abcd Medias con letras diferentes dentro de la misma fila difieren a $\mathrm{P}<0.05$ (8)

Rodríguez (16), al emplear en dietas de gallinas White Leghorn diferentes niveles de inclusión de levadura, incrementó el peso del huevo; sin embargo, en los estudios realizados por Maia et al. (17) no se observaron efecto de la levadura sobre este indicador zootécnico.

La Figura 1 muestra el porciento de huevos cascados; para 0,5 y $10 \mathrm{ml}$ de yogurt en el pienso de las aves no se presentaron diferencias significativas para este indicador zootécnico; sin embargo, entre el control y el tratamiento de inclusión de $15 \mathrm{ml}$ de yogurt en el pienso de las aves sí se presentaron diferencias significativas para $\mathrm{P}<0.05$, presentándose los porcientos más altos en el tratamiento que incluyó los $15 \mathrm{ml}$ de yogurt, lo que pudo deberse a que, con este último, al ser de mayor tamaño los huevos, es posible una disminución en el contenido de calcio, lo que a su vez influye en la debilidad del cascarón y, con ello, la mayor cantidad de huevos cascados. En este sentido, autores como Cuca (18) plantean que el calcio es uno de los elementos necesarios para el mantenimiento, producción de huevo y buena calidad del cascarón; además, este es el componente inorgánico más abundante del esqueleto y toma parte en su formación y mantenimiento, y es importante en muchas otras funciones biológicas (coagulación de la sangre, como activador y desactivador de enzimas, en la transmisión de los impulsos nerviosos y en la secreción de hormonas, entre otras).

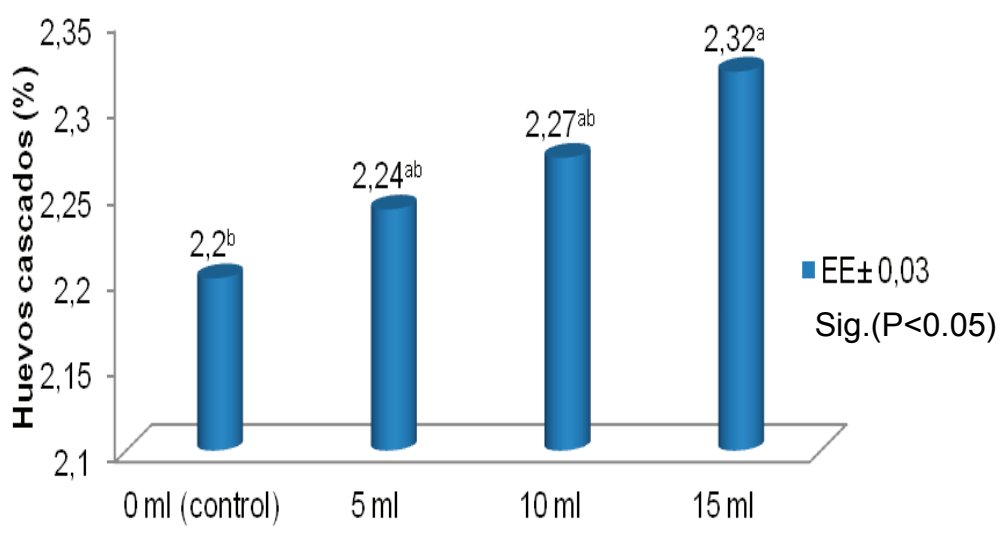

Tratamientos

${ }^{\text {ab }}$ Medias con letras diferentes difieren a $\mathrm{P}<0.05$ (8)

Fig. 1. Porciento de huevos cascados según el nivel de yogurt adicionado en el pienso. 
El $0,05 \%$ de incremento de huevos cascados en el tratamiento con $15 \mathrm{ml}$ de yogurt, con respecto al control, solo representó 4 huevos por encima del grupo control, para las 60 gallinas y los 60 días que duró el experimento en cada tratamiento, lo cual se considera irrelevante si tenemos en cuenta los 360 huevos de más que se produjeron durante toda la etapa experimental en el tratamiento con $15 \mathrm{ml}$ de yogurt con respecto al control. Sin embargo, para huevos rotos no se presentaron diferencias significativas entre los distintos niveles de inclusión del yogurt en las dietas de las gallinas Isa Brown, según se muestra en la Figura 2.

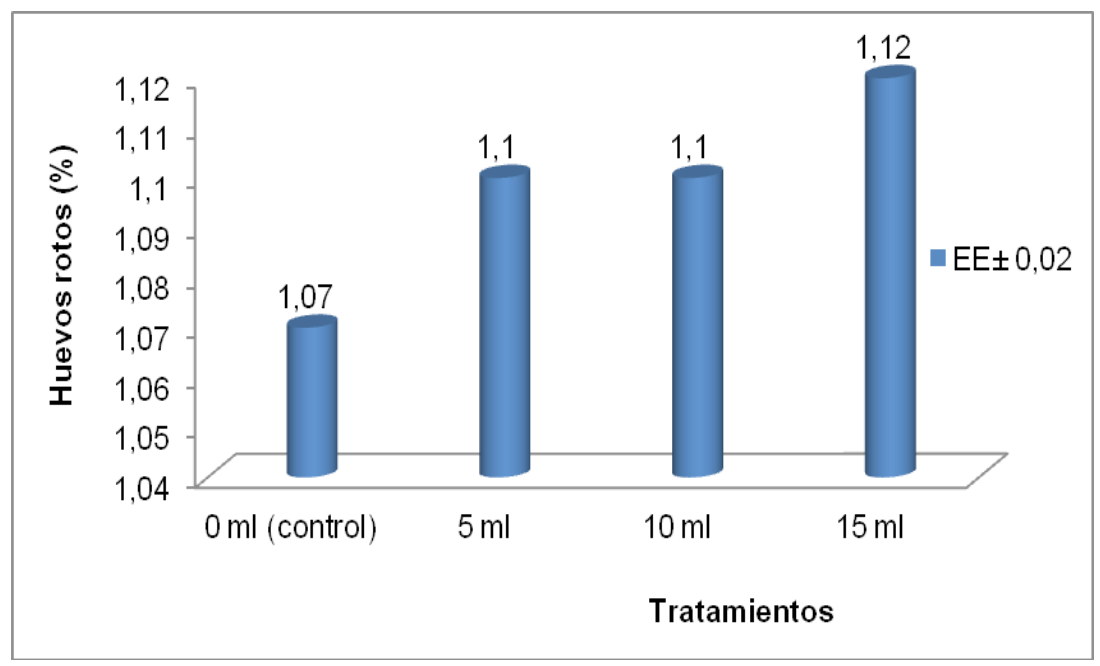

Fig. 2. Porciento de huevos rotos según el nivel de yogurt adicionado en el pienso.

Por otra parte, para los huevos sucios (Figura 3) se obtuvo que con 0,5 y $10 \mathrm{ml}$ de yogurt en el pienso de las aves no hubo diferencias significativas para este indicador zootécnico; sin embargo, los tratamientos control y $5 \mathrm{ml}$ de yogurt sí mostraron diferencias significativas para $\mathrm{P}<0.05$ con respecto a $15 \mathrm{ml}$ de yogurt. Los valores más altos de huevos sucios se observaron en el tratamiento con $15 \mathrm{ml}$ de yogurt, lo que se debió al tamaño del huevo, que al ser de mayor proporción arrastra mayor cantidad de heces contenidas en la cloaca y ocasiona pequeñas heridas sangrantes a este nivel. Este incremento representó $1.08 \%$ más de huevos sucios en el tratamiento con $15 \mathrm{ml}$ de yogurt con respecto al control, lo que significó 37 huevos por encima del grupo control para las 60 gallinas y los 60 días que duró el experimento en cada tratamiento; esta cantidad también se evalúa como poco significativa si consideramos los 360 huevos de más que se produjeron durante toda la etapa experimental en el tratamiento con $15 \mathrm{ml}$ de yogurt con respecto al control. 


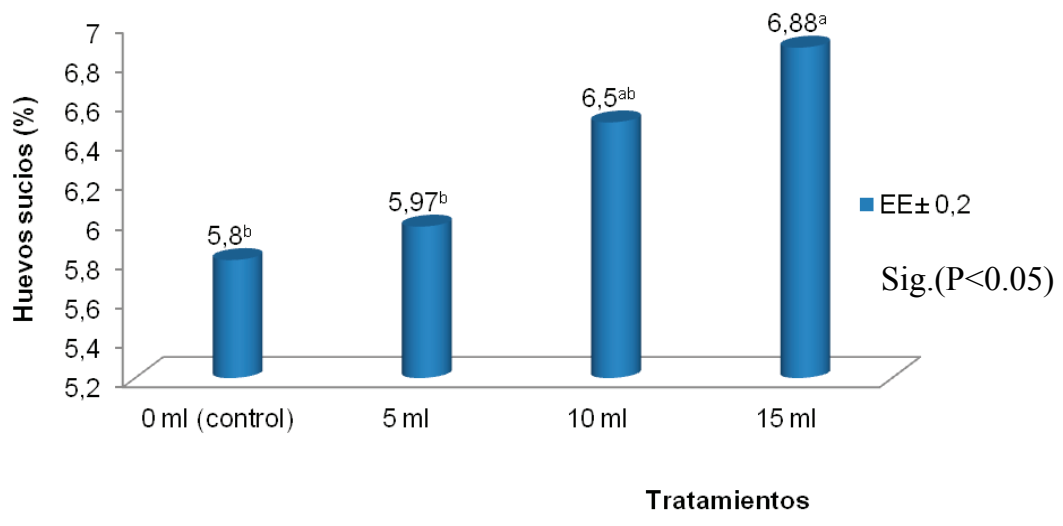

${ }^{\mathrm{ab}}$ Medias con letras diferentes difieren a $\mathrm{P}<0.05$ (8)

Fig. 3. Porciento de huevos sucios según el nivel de yogurt adicionado en el pienso.

Al analizar las características sensoriales del huevo (Tabla IV), se obtuvo que para el olor, sabor y textura no se presentaron cambios permisibles entre los cuatro tratamientos evaluados, lo que sugiere que la inclusión de yogurt artesanal elaborado con leche de búfala en dosis creciente en el pienso de gallinas ponedoras no origina cambios en las características organolépticas del huevo.

Tabla IV. Características sensoriales del huevo según el nivel de yogurt adicionado en el pienso.

\begin{tabular}{|l|l|l|l|}
\hline \multirow{2}{*}{$\begin{array}{c}\text { Nivel de yogurt en el } \\
\text { pienso/ave/día }\end{array}$} & \multicolumn{1}{|c|}{ Olor } & \multicolumn{1}{|c|}{ Sabor } & \multicolumn{1}{c|}{ Textura } \\
\cline { 2 - 4 } & Normal Anormal & Normal Anormal & Normal Anormal \\
& $\% \%$ & $\% \%$ & $\% \%$ \\
\hline $0 \mathrm{ml}$ & 1000 & 1000 & 1000 \\
\hline $5 \mathrm{ml}$ & 1000 & 1000 & 1000 \\
\hline $10 \mathrm{ml}$ & 1000 & 1000 & 1000 \\
\hline $15 \mathrm{ml}$ & 1000 & 1000 & 1000 \\
\hline
\end{tabular}

Resultados de 12 catadores encuestados.

\section{Conclusiones}

Se analizó también el color de la yema, y tampoco se observaron cambios en este indicador. Al respecto, Maia et al. (17) plantea que la pigmentación de la yema está determinada, en mayor medida, por la cantidad de pigmentos en la dieta, metabolismo de los carotenoides, deposición de carotenos en yema e intensidad del carotenoide. En este sentido, la coloración estable de la yema encontrada en el presente trabajo pudo deberse a que el ingrediente mayoritario en las dietas de las gallinas fue el maíz, cereal que aportó, en mayor medida, los pigmentos (zeaxantina y luteína) para la coloración de las yemas (19).

Se concluye que la inclusión del yogurt artesanal elaborado con leche de búfala en la dieta de gallinas ponedoras Isa Brown estimuló el índice de puesta y el tamaño del huevo, sin que se presentaran efectos significativos en la calidad sensorial del huevo.

\section{Referencias}

(1) López JR. Perspectivas de la crianza del búfalo de agua (Bubalus bubalis) en la Amazonía Ecuatoriana. Revista Amazónica Ciencia y Tecnología. 2013; 2(1): 19-29. 
(2) Hurtado T. Determinación de la calidad sanitaria y organoléptica del queso artesanal con procesamiento de la leche de búfala a partir de la pasteurización. Tesis en opción al título de Máster. Facultad Agroforestal, Departamento Agronomía, Universidad de Guantánamo. Cuba. 2015.

(3) Metchnikoff E. The prolongation of life. En: Optimistic studies. Heinemann, William. Ed. G. P. Putnam \& Sons, London, UK 1907, 1-100.

(4) Carro MD, Ranilla MJ. Aditivos antibióticos promotores del crecimiento de los animales: Situación actual y posibles alternativas. Departamento de Producción Animal Universidad de León. 2005.

(5) Pérez G. Una nueva herramienta profiláctica: "La exclusión competitiva en avicultura". Venezuela Avícola. 2005; 11(3): 25-33.

(6) Rodríguez O., Martín Y., García A., Núñez M. Suero fermentado por lactobacillus casei y lactobaciIlus acidophilus con propiedades probióticas para cerdos jóvenes. En: Memorias del V Congreso de Producción Animal Tropical. Palacio de las Convenciones, La Habana, Cuba 16 al 20 de noviembre de 2015.

(7) Medina R., Ortiz A., Elías A. Efecto del probiótico Biolac sobre parámetros productivos y de salud en la categoría de precebas en convenios porcinos. En: Memorias del V Congreso de Producción Animal Tropical. Palacio de las Convenciones, La Habana, Cuba 16 al 20 de noviembre de 2015.

(8) Duncan DB. Multiple ranges and multiple F test. Biometrics, 1955; 11(1): 1-42. DOI: http://doi. org/10.2307/3001478.

(9) IBM Corporation. Statistical Package for Social Sciences. New York. 2009.

(10) Font H., Noda A., Torres V., Herrera M., Lizazo D., Sarduy L., Rodríguez L. Programa Comparpro 1.0. Departamento de Biomatemática. Instituto de Ciencia Animal. La Habana, Cuba. 2007.

(11) Schreiner M., Howard WH, Razzazi-Fazeli E., Böhm J., Moreira RG. Effect of different sources of dietary omega-3 fatty acids on general performance and fatty acid profiles of thigh, breast, liver and portal blood of broilers. I Sci Food Agric. 2005; 85: 219-226. DOI: http://doi.org/10.1002/jsfa.1948.
(12) Rondón AJ. Obtención de biopreparados a partir de lactobacillus autóctonos de tracto digestivo de pollo y evaluación de su efecto probiótico en estos animales. Tesis presentada en opción al grado científico de Doctor en Ciencias Veterinarias. Departamento de Fisiología, Instituto de Ciencia Animal (ICA). Mayabeque, Cuba. 2009.

(13) García Y. Obtención de microorganismos con actividad probiótica a partir de excretas de pollos de ceba fermentadas. Tesis presentada en opción al grado científico de Doctor en Ciencias Veterinarias. Departamento de Fisiología, Instituto de Ciencia Animal (ICA). Mayabeque, Cuba. 2011.

(14) Yoo IK, Chang HN, Lee EG, Chang YK, Moon SH. Effect of B vitamin supplementation on lactic acid production by $L$. casei. J. Fermentation Bioeng. 1997; 84: 172-175. DOI: http://doi.org/10.1016/ S0922-338X(97)82551-2.

(15) Gómez G., Balcázar JL. A. review on the interactions between gut microbiota and innate immunity of fish. FEMS Immun. Medical Microbiol. 2008; 52: 145-154. DOI: http://doi.org/10.1111/j.1574695X.2007.00343.x.

(16) Rodríguez B. Levadura torula desarrollada sobre vinaza de destilerías para la alimentación de aves. Tesis presentada en opción al grado científico de Doctor en Ciencias Veterinarias. Departamento de Animales Monogástricos, Instituto de Ciencia Animal (ICA). Mayabeque, Cuba. 2011.

(17) Maia GA, Fonseca JB, Soares RT, Silva MA. Desempenho de poedeiras comerciais alimentadas com levedura seca (Saccharomyces cerevisiae) de cana de açucar. Rev. Bras. Cienc. Avic. 2001; 3(2): 1-9. DOI: http://doi.org/10.1590/S1516635X2001000200005.

(18) Cuca M. Estudios recientes con calcio en gallinas de postura. (en línea) 2013 (fecha de acceso 12 abril de 2015) Disponible en: http://www.engormix.com/MA-avicultura/nutricion/articulos.

(19) Martínez Y. Alimentación de gallinas ponedoras y pollos de ceba con harina de semillas de calabaza. Tesis presentada en opción al grado científico de Doctor en Ciencias Veterinarias. Departamento de Animales Monogástricos, Instituto de Ciencia Animal (ICA). Mayabeque, Cuba. 2009. 